\title{
Determinants of white-coat hypertension
}

\author{
E. Dolan ${ }^{a}$, A. Stanton ${ }^{a}$, N. Atkins ${ }^{a}$, E. Den Hond ${ }^{b}$, L. Thijs ${ }^{b}$, P. McCormack ${ }^{a}$, \\ J. Staessen ${ }^{\mathrm{b}}$ and E. O'Brien ${ }^{\mathrm{a}}$
}

\begin{abstract}
Background The prevalence of white-coat hypertension (WCH) is considerable in patients referred with elevated office blood pressure. Failure to recognise this phenomenon can lead to the inappropriate use of antihypertensive medications. We undertook this study to determine the profile of patients with $\mathrm{WCH}$.
\end{abstract}

Methods Baseline clinic and daytime ambulatory blood pressures were available from $\mathbf{5 7 1 6}$ patients referred over a 22-year period. Individuals were considered to have $\mathrm{WCH}$ if they had an elevated clinic blood pressure measurement greater than $140 / 90 \mathrm{mmHg}$ and normal daytime mean ambulatory blood pressure. Mean age was $\mathbf{5 3 . 6}$ years and $53.2 \%$ were female.

Results The overall prevalence of white-coat hypertension was $15.4 \%$. A higher prevalence was seen amongst older adults, females, and non-smokers.

\section{Introduction}

Blood pressure measurements in the clinical setting may not reflect blood pressure behaviour away from the medical environment [1]. White-coat hypertension (WCH) is the most commonly used term to describe individuals whose blood pressure is only high in the medical setting. Twenty-four-hour ambulatory blood pressure monitoring (ABPM) by providing frequent readings during routine daily activities gives an accurate assessment of blood pressure behaviour, [2] and the technique is now recognized as the most effective means of detecting WCH. Guidelines on hypertension list WCH as one of the few indications for ABPM [3-5]. White-coat hypertension has a relatively benign outcome compared to sustained mild hypertension, which means that failure to recognize the condition may lead to the inappropriate use of antihypertensive medications [6,7]. Unfortunately, patients with WCH have few characteristics other than an elevated blood pressure in the medical environment to assist in suspecting the condition so as to allow greater selection for ABPM. We undertook this study to evaluate the determinants of $\mathrm{WCH}$ in a referred population.

\section{Methods}

\section{Study population}

This study analysed blood pressure data of patients referred to the Blood Pressure Unit since 1 June 1980. For this analysis, we selected all patients who had both clinic

\begin{abstract}
Conclusion Multivariate logistic regression analysis confirmed these characteristics as independent predictors of WCH. Blood Press Monit 9:307-309 (c) 2004 Lippincott Williams \& Wilkins.
\end{abstract}

Blood Pressure Monitoring 2004, 9:307-309

Keywords: white-coat hypertension, ambulatory blood pressure measurement, clinic blood pressure measurement, clinical characteristics

aADAPT Centre, Beaumont Hospital, and Department of Clinical Pharmacology, Royal College of Surgeons in Ireland, Dublin, Ireland and ${ }^{\mathrm{b}}$ Katholieke Universiteit Leuven, UZ Gasthuisberg, Leuven, Belgium.

Correspondence and requests for reprints to Professor E. O'Brien, ADAPT Centre, Beaumont Hospital, Dublin 9, Ireland.

E-mail: eobrien@iol.ie

Received 24 August 2004 Accepted 24 September 2004

and ABPM available from their initial visit, along with other baseline characteristics (smoking history and BMI). A total of 5716 patients referred because of elevated clinic blood pressure, who had been instructed to stop antihypertensive medication prior to the initial visit, were eligible for inclusion.

\section{Ambulatory blood pressure measurement}

Ambulatory measurements were made every half-hour throughout the 24-h period using SpaceLabs 90202 and 90207 monitors (SpaceLabs Inc., Redmond, Washington, USA; SpaceLabs Berkshire, UK), which have previously been validated $[8,9]$. All data were transferred into a specialised software package, $\left(\right.$ dabl $^{\circledR}$ ABPM, DABL Limited, Dublin, Ireland) [10] allowing calculation of systolic and diastolic blood pressures and heart rate in the white-coat window (maximum reading in the first hour recording), daytime pressures (average of readings between 0900 and $2100 \mathrm{~h}$, with the white-coat window excluded), and night-time pressures (average of readings between 0100 and $0600 \mathrm{~h}$ ) [11]. Ambulatory blood pressure monitoring recordings were regarded as satisfactory if at least 14 daytime readings and six night-time readings were available.

\section{Clinic blood pressure measurement}

The attending nurse in the Blood Pressure Unit performed clinic blood pressure measurement, when 
the patient arrived for ABPM. The mean of three clinic measurements was calculated in the non-dominant arm after $5 \mathrm{~min}$ of quiet sitting in accordance with the recommendations of the British Hypertension Society [5], using either a standard mercury sphygmomanometer or a the automated Omron HEM-705CP, which has been validated [12].

\section{Diagnosis of white-coat hypertension}

The definition of WCH used in this study was in accordance with European Society of Hypertension guidelines [4], namely a clinic blood pressure greater than $140 / 90 \mathrm{mmHg}$ and daytime mean ABPM less than $135 / 85 \mathrm{mmHg}$.

\section{Statistical analysis}

SAS software (SAS Institute, Cary, North Carolina, USA), version 8.1 was used for statistical analysis. Data are reported as mean \pm standard deviation. In univariate analysis, proportions were compared by the chi-squared test. In multivariate analysis, a multiple logistic regression analysis model was used. Gender, age, body mass index, smoking history and clinic systolic blood pressure were entered into this model and considered as possible independent determinants of WCH. $P$ values $<0.05$ were considered statistically significant.

The approval of the local Hospital Ethics Committee was obtained and the management of all data was in keeping with best practice guidelines

\section{Results}

\section{Patient characteristics}

A total of 5716 patients were included in the study of which $53.2 \%$ were female. Mean age was 53.6 years with a range of $17-92$ years. In all, $23.9 \%$ were current smokers, and body mass index averaged $27.3 \mathrm{~kg} / \mathrm{m}^{2}$. Females had higher clinic systolic blood pressure but lower mean daytime mean systolic ambulatory pressures (Table 1 ).

\section{Determinants of WCH}

The overall prevalence of WCH in the study cohort was $15.4 \%$. Older patients had a higher prevalence of WCH (Table 2). There was a higher prevalence of WCH among non-smokers, females, and patients with lower clinic systolic blood pressure had a higher prevalence of WCH. Logistic regression analysis showed that age $(P<0.01)$, gender $(P<0.001)$, and smoking history $(P<0.001)$ were independent predictors of WCH.

\section{Discussion}

Suspected WCH is an established indication for ABPM in a number of hypertension guidelines [3-5]. Understanding the likely profile of individuals with the condition could assist in the selection of patients for ABPM. Only a few studies have evaluated the probability of WCH based
Table 1 Baseline characteristics according to gender

\begin{tabular}{lccc}
\hline & All & Male & Female \\
\hline$n$ & 5716 & 2676 & 3040 \\
Age (years) & $53.6(14.9)$ & $52.4(14.5)$ & $54.6(15.1)$ \\
BMl (kg/m $\left.{ }^{2}\right)$ & $27.3(4.9)$ & $27.5(4.1)$ & $27.1(5.5)$ \\
Current smokers $(\%)$ & 23.9 & 25.2 & 22.8 \\
Clinic SBP & $162.0(27.7)$ & $160.8(27.3)$ & $163.1(28.9)$ \\
Clinic DBP & $93.0(14.8)$ & $93.8(15.2)$ & $92.4(14.4)$ \\
Day SBP & $145.9(18.7)$ & $147.3(16.6)$ & $144.8(18.7)$ \\
Day DBP & $88.8(12.6)$ & $90.9(12.9)$ & $86.9(12.0)$ \\
Night SBP & $128.6(20.0)$ & $129.6(19.2)$ & $127.8(20.6)$ \\
Night DBP & $75.0(12.8)$ & $77.2(13.0)$ & $73.2(12) .4$ \\
\hline
\end{tabular}

Mean values (standard deviation). BMI, body mass index. SBP, systolic blood pressure, DBP, diastolic blood pressure.

Table 2 Prevalence of white-coat hypertension (WCH) according to patient characteristics

\begin{tabular}{lcrcc}
\hline Characteristic & Grouping & $n$ & $\begin{array}{c}\text { Percentage } \\
\text { with WCH } \\
(\%)\end{array}$ & P value \\
& & & & \\
\hline Gender & Female & 3040 & 16.8 & $<0.001$ \\
Age & Male & 2676 & 13.7 & \\
& $<35$ years & 682 & 12.1 & $<0.001$ \\
& $35-50$ years & 1574 & 14.3 & \\
Current smoking & $50-65$ years & 2194 & 16.4 & \\
& $>65$ years & 1266 & 16.9 & \\
Body mass index & No & 4348 & 16.7 & $<0.001$ \\
& Yes & 1366 & 11.3 & \\
& $<25\left(\mathrm{~kg} / \mathrm{m}^{2}\right)$ & 2096 & 15.9 & NS \\
Clinic SBP & $25-29.9\left(\mathrm{~kg} / \mathrm{m}^{2}\right)$ & 2271 & 15.4 & \\
& $>30\left(\mathrm{~kg} / \mathrm{m}^{2}\right)$ & 1399 & 15.1 & \\
& $140-159 \mathrm{mmHg}$ & 1497 & 31.2 & $<0.001$ \\
& $160-170 \mathrm{mmHg}^{2}$ & 1543 & 18.7 & \\
& $<180 \mathrm{mmHg}$ & 1521 & 11.8 & \\
\hline
\end{tabular}

$P$ values relate to chi-squared test. SBP, systolic blood pressure.

on the clinical characteristics of subjects with the condition and some of these have shown conflicting results [13]. Female sex, young age and brief known duration of hypertension were among independent predictors of WCH in a study carried out in 292 subjects with office diastolic blood pressure between 90 and $104 \mathrm{mmHg}$ [14]. In an international database of 2492 subjects with clinic blood pressure greater than $140 \mathrm{mmHg}$ systolic and/or $90 \mathrm{mmHg}$ diastolic, the probability that hypertensive subjects had an average 24-h ABPM below the 95th centile of a normotensive control group was greater among women and older adults [15].

In our large cohort of patients referred for further evaluation of elevated clinic blood pressures, age, smoking history and gender were independent predictors of WCH. In keeping with other studies WCH was present in a significant proportion of these patients - more than $15 \%$, and nearly a third of patients with clinic systolic blood pressures between $140-159 \mathrm{mmHg}$ had WCH $[6,14]$. Given that the cohort was taken from a population referred to a hypertensive clinic, the prevalence of WCH observed is probably higher than in a normal population. Also a more conservative cut-off for daytime mean ABPM, as used in other studies, would have reduced the observed prevalence [16]. 
The findings of this study emphasize the importance of using ABPM in the initial evaluation of patients referred for assessment of elevated clinic blood pressure, particularly in older patients, non-smokers and females.

\section{References}

1 Mancia G, Grassi G, Pomidossi G, Gregorini L, Bertineri G, Parati G, et al. Effects of blood pressure measurement by the doctor on patient's blood pressure and heart rate. Lancet 1983; 2: 695-698.

2 Pickering TG. Blood pressure measurement and detection of hypertension. Lancet 1994; 344:31-35.

3 Chobanian AV, Bakris GL, Black HR, Cushman WC, Green LA, Izzo JL. Jr, et al. The seventh report of the Joint National Committee on Prevention, Detection, Evaluation, and Treatment of High Blood Pressure: the JNC 7 Report. JAMA 2003; 289:2560-2571.

4 O'Brien E, Asmar R, Beilin L, Imai Y, Mallion J-M, Mancia G, et al., on behalf of the European Society of Hypertension Working Group on Blood Pressure Monitoring. European Society of Hypertension recommendations for conventional, ambulatory and home blood pressure measurement. J Hypertens 2003; 21:821-848.

5 Williams B, Poulter NR, Brown MJ, Davis M, Mclnnes GT, Potter JF, et al. British Hypertension Society: guidelines for management of hypertension. Report of the fourth working party of the British Hypertension Society, 2004BHS IV. J Hum Hypertens 2004; 18:139-185.

6 Khattar RS, Senior R, Lahiri A. Cardiovascular outcome in white-coat versus sustained mild hypertension: a 10-year follow-up study. Circulation 1998; 98:1892-1897.

7 Celis H, Staessen JA, Thijs L, Buntinx F, De Buyzere M, Den Hond E, et al. Ambulatory Blood Pressure and Treatment of Hypertension Trial
Investigators, Cardiovascular risk in white-coat and sustained hypertensive patients. Blood Press Monit 2002; 11:352-356.

8 O'Brien E, Sheridan J, Browne T, Conroy R, O'Malley K. Validation of the SpaceLabs 90202 ambulatory blood pressure recorder. J Hypertens 1989; 7(Suppl 6):S388-S389.

9 O'Brien E, Mee F, Atkins N, O'Malley K. Accuracy of the SpaceLabs 90207 determined by the British Hypertension Protocol. J Hypertens 1991; 9 : 573-574.

10 Atkins N, Mee F, O'Brien E. A customized international database system for storing and analysing ambulatory blood pressure measurements and related data. Abstract. J Hypertens 1994; 12:S23.

11 Owens P, Atkins N, O'Brien E. Diagnosis of white-coat hypertension by ambulatory blood pressure monitoring. Hypertension 1999; 34:267-272.

12 O'Brien E, Mee F, Atkins N, Thomas M. Evaluation of three devices for selfmeasurement of blood pressure according to the revised British Hypertension Society Protocol: the Omron HEM-705CP, Phillips HP5332, and Nissei DS-175. Blood Press Monit 1996; 1:55-61.

13 Verdecchia P, O'Brien E, Pickering T, Staessen JA, Parati G, Myers M, et al. European Society of Hypertension Working Group on Blood Pressure Monitoring. When can the practicing physician suspect white-coat hypertension? Statement from the Working Group on Blood Pressure Monitoring of the European Society of Hypertension. Am J Hypertens 2003 16:87-91.

14 Pickering TG, James GD, Boddie C, Harshfield GA, Blank S, Laragh JH. How common is white-coat hypertension? JAMA 1988; 259:225-228.

15 Staessen JA, O'Brien ET, Atkins N, Amery AK. Short report: ambulatory blood pressure in normotensive compared with hypertensive subjects The Ad-Hoc Working Group. J Hypertens 1993; 11:1289-1297.

16 Verdecchia P, Palatini P, Schillaci G, Mormino P, Porcellati C, Pessina AC. Independent predictors of isolated clinic ('white-coat') hypertension. J Hypertens 2001; 19:1015-1020. 